\title{
Crystal Linear and Circular Dichroism of the Copper(II) Complex of the Schiff Base from R-1,2-Propanediamine and Acetylacetone
}

\author{
HANS PETER JENSEN *
}

Institute of Molecular Biology, University of Oregon, Eugene, Oregon 97403, U.S.A.

The crystal LD and CD spectra in the visible range have been studied for the copper(II) complex of the Schiff base from $R$-1,2-propanediamine and two molecules of acetylacetone. The results have been used to assign possible ligand field transitions to absorption bands of known polarizations. It is shown that the dipole strength of a given absorption band need not have the same origin as a whole, as part of the absorption in the visible range can be accounted for by $d_{y x} \rightarrow d_{x y}$ and $d_{y x} \rightarrow \pi^{*}$ transitions. Furthermore, the instrumentation used to obtain LD and CD spectra of an anisotropic crystal is discussed.

Much work has been devoted to gain insight into the crystalline, molecular and electronic structure of copper(II) complexes of $\boldsymbol{\beta}$-diketones and of Schiff base derivatives from $\beta$-diketones. $E . g$. , the crystalline structure of the anhydrate, the hemihydrate, and the monohydrate of the copper(II) complex with 4,4' - (ethylenediimino)di(3-pentene-2-one) [Cu en(acac $\left.)_{2}\right]$ was determined a number of years ago ${ }^{1-3}$ and recently the crystalline structure of the copper(II) complex of 4,4' - $(R$-1,2-propanediimino)di(3-pentene-2-one) [Cu Rpn(acac) $)_{2}$ has been determined.*

By studying absorption and circular dichroism (CD) spectra of $\mathrm{Cu} \mathrm{Rpn}(\mathrm{acac})_{2}$, it has been concluded that this type of compound in solution has a structure somewhere between tetrahedral and planar arrangements; ${ }^{5,6}$ in this specific case it was also possible to give the chiralty of the dominating structure later confirmed by the X-ray structural analysis."

* On leave from Chemistry Department A, Technical University of Denmark, DK-2800 Lyngby, Denmark.

Acta Chem. Scand. A 30 (1976) No. 2
Recently it has been shown from linear dichroism (LD) and CD measurements that the nonplanar structure of $\mathrm{Cu}$ en(acac) $)_{2}$ observed in the crystalline structure is also present in solution. ${ }^{7}$ While there is a fair degree of consistency among structural assignments for this type of compound, the situation becomes a little more confused with respect to electronic configuration and assignments to absorption bands in the visible and near ultraviolet (UV) range. Extended Hückel calculations on the molecular orbitals in bis( $\beta$-ketoenolate) complexes of copper(II) assuming a planar structure of the compounds indicated the relative energies of the ligand $\pi$-orbitals and the metal ion $3 d$-orbitals to be $3 \pi \approx 3 d<4 \pi$ with $3 d_{x y}$ as the half-filled orbital in the metal $3 d^{9}$-system. ${ }^{8}$ It seems to be generally accepted that $3 d_{x y}$ is the high energy orbital of the metal ion even when these complexes are nonplanar and twisted towards a tetrahedral structure,,$^{5,6,9}$ but when it comes to assignments of the electronic transitions in the ligand field range, certain ambiquities are evident. There are three bands of interest for the complexes $\mathrm{Cu}$ en(acac) $)_{2}$ and $\mathrm{Cu} \operatorname{Rpn}(\mathrm{acac})_{2}, \quad \mathrm{a}$ shoulder at $\sim 16000 \mathrm{~cm}^{-1}$ (I) on the main peak in the visible at $\sim 18000 \mathrm{~cm}^{-1}$ (II) and a band hidden in the foot of the internal ligand $\pi \rightarrow \pi^{*}$ transitions at $\sim 22000-23000 \mathrm{~cm}^{-1}$ (III). The earlier assignments to these bands have been summarized in Table 1 .

Some of the ambiguity stems from the fact that different approaches have been used in the papers cited. The argument of Downing and Urbach for II being $d_{z^{2} \rightarrow d_{x y}}$ is based on the experimental fact that II is shifted rather 


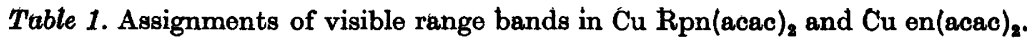

\begin{tabular}{|c|c|c|c|c|c|c|}
\hline Compound & Band & Assignment & $\begin{array}{l}\text { Treated in } \\
\text { point group a }\end{array}$ & $\begin{array}{l}\text { Polari- } \\
\text { zation } b\end{array}$ & Ref. & Phase \\
\hline Cu Rpn(acac) & $\begin{array}{l}\text { I } \\
\text { III }\end{array}$ & $\begin{array}{l}\left(x^{2}-y^{2}\right) \rightarrow(x y) \\
\left(z^{2}\right) \rightarrow(x y) \\
(x z) \rightarrow(x y)\end{array}$ & $\begin{array}{l}C_{2 v} \\
C_{2 v} \\
C_{2}\end{array}$ & $\begin{array}{l}y \\
y \\
x\end{array}$ & 5 & solution \\
\hline Cu Rpn(acac) & $\begin{array}{l}\text { I } \\
\text { II } \\
\text { III }\end{array}$ & $\begin{array}{l}\left(x^{2}-y^{2}\right) \rightarrow(x y) \\
(x z) \rightarrow(x y) \\
(x y) \rightarrow 4 \pi \\
\text { or }\end{array}$ & $\begin{array}{l}D_{2} \\
D_{2} \\
D_{2}\end{array}$ & $\begin{array}{l}z \\
x \\
x \text { and } z\end{array}$ & 6 & solution \\
\hline $\mathrm{Cu}$ en $(\mathrm{acac})_{2}$ & $\begin{array}{l}\text { I } \\
\text { II } \\
\text { III }\end{array}$ & $\begin{array}{l}n \rightarrow \pi^{*} \\
\left(x^{2}-y^{2}\right) \rightarrow(x y) \\
\left(z^{2}\right) \rightarrow(x y) \\
\text { not a } d \rightarrow d\end{array}$ & $\begin{array}{l}D_{2} \\
C_{2 v} \\
C_{2 v}\end{array}$ & $\begin{array}{l}x \text { and } z \\
y \\
y\end{array}$ & 9 & crystalline \\
\hline
\end{tabular}

a The point group of $\mathrm{Cu}$ diamine (acac) $)_{2}$ cannot be higher than $C_{2}$; on the other hand, one can obtain a better group to work in if one either takes the deviation from planarity to be small $\left(C_{2 v}\right)$ or puts the $\mathrm{N}$ and $O$ equal and forgets the ethylene bridge $\left(D_{2}\right){ }^{b}$ Polarization direction in an orthogonal molecular coordinate system where (a), the ligators define the $x y$-plane (least mean squares); (b), the $x$-axis is the projection in this plane of the line passing the midpoints between the two oxygens and the two nitrogens; (c), the z-axis is normal to the $x y$-plane and passes through the metal ion; see fig. 2 of Ref. 6.

much by adding pyridine to the complex in solution. The assignments of Jensen and Larsen are based on interpretations of CD spectra assuming that the $d \rightarrow d$ transitions "borrow" electric transition dipole moments from the internal ligand $\pi \rightarrow \pi^{*}$ transitions, and the assignments of Olson et al. come from a study of the polarized crystal spectra interpreted in an oriented gas model. From the crystal structure of $\mathrm{Cu}$ Rpn(acac) ${ }_{2}^{*}$ it is seen that the molecules in the unit cell are oriented in such a way that measurements with linearly polarized light along the a and $c$ axes would give rise to very weak absorptions of $y$-polarization in the molecular coordinate system (Table 2). Therefore in the case of $\mathrm{Cu} \mathrm{Rpn(acac})_{8}$ it is possible to study the $x$-and $z$-polarized bands specifically contrary to Olson et al. dealing with $\mathrm{Cu}$ en(acac) $)_{\mathbf{2}}$.

\section{SPECTRAL PROPERTIES OF ANISOTRO. PIC CRYSTALS}

A crystal of $\mathrm{Cu} \mathrm{Rpn}(\mathrm{acac})_{2}$ is an anisotropic medium capable of showing LD because of the orientation of the molecules in the crystal and CD because of the molecular asymmetry. Also, because of the Kronig-Kramers relationships, linear birefringence (LB) and circular birefringence (CB) have to be taken into consideration. Consequently, the linearly polarized light used in measuring polarized absorption spectra of an oriented crystal of, e.g., Cu en(acac), (without molecular asymmetry) will generally be subjected to both LD and LB effects. The crystal may be considered as a stack of slices of infinitesimal pathlengths, ${ }^{10}$ and the linearly polarized light in slice 1 will then be subjected to a LD effect associated with a rotation, $\alpha_{1}$,

Table 2. Squares of direction cosines in $\mathrm{Cu} \mathbf{R p n}(\mathrm{acac})_{2}$ crystal.

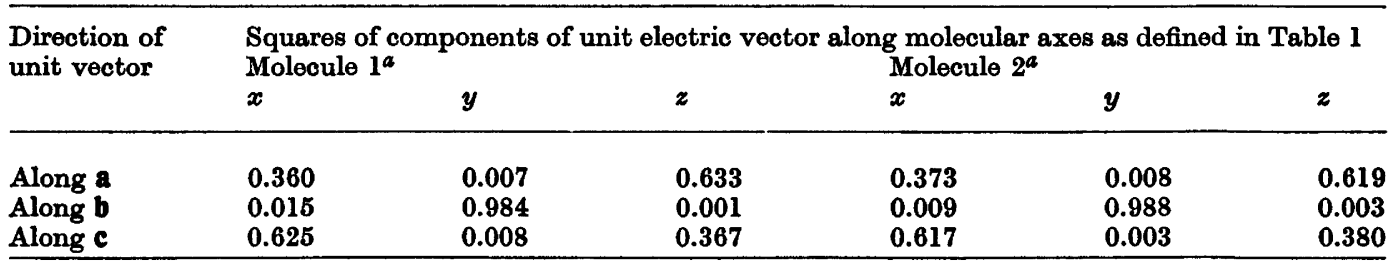

a Orthorhombic crystal $P 2_{1} 2_{1} 2_{1}$ with four equivalent positions, every position has two molecular units. 
of the vibration direction; therefore the polar. ized absorption in slice 2 is measured in a different direction than in slice 1 . In a crystal of $\mathrm{Cu} \mathrm{Rpn}(\mathrm{acac})_{2}$, this might get even worse since furthermore we have a CB effect which also has a rotation of the vibrational direction of linearly polarized light as result. Thus it turns out that we have to consider the influence of all the possible effects of the anisotropic crystal on polarized light in designing the experiment by which we will determine the specific effects. A way of handling this problem is shown by $G^{1} 11$ who demonstrates that in writing the incoming light as a Stokes vector, the different effects of a general media can be expressed in a $4 \times 4$ Mueller matrix $F(l)$ which again is related to another $4 \times 4$ Mueller matrix H through the relation

$\mathbf{F}(l)=\exp (-2 \eta l \mathbf{H})$

where $\eta$ is the number density of active molecules (in this case $\eta=1$ ) and $l$ is the pathlength. The elements in the $\mathbf{H}$ matrix have been related to the phenomenological parameters $\mathrm{LD}, \mathrm{LB}$, CD, and CB by Troxell and Scheraga.12

\section{INSTRUMENTATION}

As the mathematical apparatus for handling the effects of a general anisotropic medium on light is available, the subject will now be the selection of a particular effect by instrumentation. A phase modulation spectrophotometer capable of selecting any one of the four effects (LD, LB, CD, CB) has been described in principle by Hofrichter and Schellman, ${ }^{13}$ and we shall only briefly describe the instrumentation for measuring $\mathrm{LD}$ and $\mathrm{CD}$ on microscopic samples.

Light from a double monochromator passes through a polarizer and then through an optical modulator with principal axes at $45^{\circ}$ to that of the polarizer. The modulator ${ }^{14}$ is a piece of fused silica, which is driven to oscillation by mechanical coupling to a quartz crystal cut so that it has a resonance frequency of $50 \mathrm{kHz}$. The oscillation is selected so that it provides a periodically varying birefringence in the silica plate with an amplitude that is determined by the strength of the signal from the driving oscillator. Light which is polarized $45^{\circ}$ to the axes of a birefringent plate emerges with a variety of polarizations depending on the magnitude of the birefringence. If the phase difference introduced by the modulator sweeps back and forth between $-\pi / 2$ and $+\pi / 2$, the light switches between the two orthogonal forms of circularly polarized light with the same frequency as the oscillator $(50 \mathrm{kHz})$ and if the phase difference sweeps between $-\pi$ and $+\pi$, the light switches between the two orthog. onal forms of linearly polarized light at twice the frequency of the oscillator $(100 \mathrm{kHz})$.

After leaving the modulator, the light passes through the sample which is centered in the light beam on the stage of a microscope and with the image of the sample focused at the photomultiplier position. The photocurrent is then sent through a lock-in-amplifier, selecting either a $50 \mathrm{kHz}$ or $100 \mathrm{kHz}$ AC current. To calculate the light intensity proportional to the photocurrent, the following procedure may be used:

$s_{0}=F_{\text {medium }} F_{\text {modulatior }} F_{\text {polarizer }} S_{i}$

where $s_{i}$ and $s_{o}$ are the Stokes vectors of the incoming and outgoing light beam, respectively, and the F's Mueller matrices. ${ }^{15}$ It should be noted that $\mathbf{F}_{\text {medium }}$ from Troxell and Scheraga is arranged to match a Stokes vector developed on basis of Pauli spin matrices. ${ }^{16}$ Multiplying the matrices together determines the output intensity (matrix-element 1 in $s_{o}$ ). The result has terms containing $\cos \left(\delta_{\max } \sin \omega t\right)$ and $\sin$ $\left(\delta_{\max } \sin \omega t\right)$ due to modulation. These may be expanded in Fourier series:

$\cos \left(\delta_{\max } \sin \omega t\right)=J_{0}\left(\delta_{\max }\right)+$

$2 \sum_{k=1}^{\infty} J_{2 k}\left(\delta_{\max }\right) \cos 2 k \omega t$

$\sin \left(\delta_{\max } \sin \omega t\right)=2 \sum_{k=1}^{\infty} J_{2 k-1}\left(\delta_{\max }\right) \sin (2 k-1) \omega t$

where $J_{n}$ are Bessel functions of order $n$.

For the LD and CD effects keeping only DC, $\omega$, and $2 \omega$ terms and using only the expansion of $\mathbf{H}$ to the second order, then

$I$ (photocurrent) $\propto \frac{1}{2} e^{-\mathrm{Abs}}\left[1+\frac{1}{2}\left(\mathrm{CD}^{2}+\mathrm{LD}^{2}\right)-\right.$

$\mathrm{LD} J_{0}\left(\delta_{\max }\right)+2 \mathrm{CD} J_{1}\left(\delta_{\max }\right) \sin \omega t-$

$\left.2 \mathrm{LD} J_{2}\left(\delta_{\max }\right) \cos 2 \omega t\right]$

To detect LD, a modulation of $\pi \sin \omega t$ (maxi-

Acta Chem. Scand. A 30 (1976) No. 2 
mizes $J_{\mathrm{a}}$ ) should be used and signals at $2 \omega$ (100 kHz) should be observed, thus:

$$
\frac{\left\langle I_{\mathrm{AC}}\right\rangle}{I_{\mathrm{DC}}} \propto \frac{-2 \mathrm{LD} J_{2}\left(\delta_{\max }\right)}{1+\frac{1}{2}\left(\mathrm{CD}^{2}+\mathrm{LD}^{2}\right)-\mathrm{LD} J_{0}\left(\delta_{\max }\right)}
$$

To detect CD a modulation of $\pi / 2 \cdot \sin \omega t$ (maximizes $\left.J_{1}\right)$ should be used and signals at $\omega(50$ $\mathrm{kHz}$ ) should be observed, thus:

$$
\frac{\left\langle\mathrm{I}_{\mathrm{AC}}\right\rangle}{I_{\mathrm{DC}}} \propto \frac{2 \mathrm{CD} J_{1} \delta\left(_{\max }\right)}{1+\frac{1}{2}\left(\mathrm{CD}^{2}+\mathrm{LD}^{2}\right)-\mathrm{LD} J_{0}\left(\delta_{\max }\right)}
$$

As may be seen from these expressions it is important to keep $\operatorname{LD}(\lambda)=0$ when measuring $\mathrm{CD}(\lambda)$ in case $\mathrm{LD}>\mathrm{CD}$.

\section{EXPERIMENTAL}

Cu Rpn(acac) $)_{2}$ and $\mathrm{Cu}$ en(acac) $)_{2}$ were prepared and crystals grown as previously described.4, The identity of the compounds was established from chemical analysis. Suitable crystals (app. size $0.5 \mathrm{~mm} \times 0.5 \mathrm{~mm} \times 0.1 \mathrm{~mm}$ ) were glued to cover glasses and the position of the unit cell axes in the tabular faces of the crystals was determined by the Buerger precession method. The signs of the LD curves as well as the absorption scales were established by using a standard made from pseudoisocyanin- $N, N^{\prime}$-diethyl iodide in a stretched PVA-film.17 The sign and absorption scale of the CD curve was established by using a solution of $\mathrm{Cu} \mathrm{Rpn}(\mathrm{acac})_{2}$ as standard, furthermore the CD curve of the crystalline material was measured under the condition $\operatorname{LD}(\lambda)=0$ maintained by turning the microscope stage during the measurement.

The spectral response of the detector system (an E.M.I. 6256S PM tube mounted in a PAR model PM tube housing) is negligible above $610-620 \mathrm{~nm}$.

\section{RESULTS}

The LD curves and CD curve of $\mathrm{Cu} R p n$ (acac), and the $\mathrm{LD}$ curve of $\mathrm{Cu}$ en(acac) ${ }_{2}$ are given in Figs. 1, 2 and 3, respectively. Looking at the $\mathrm{LD}$ of $\mathrm{Cu} \mathrm{Rpn}$ (acac) ${ }_{2}$ and refering to Table 2, it is seen that the band at $620 \mathrm{~nm}$ has $x$-polarization and the band at $540 \mathrm{~nm}$ has $z$-polarization in the molecular coordinate system.

The crystal CD of $\mathrm{Cu} R \mathrm{Rp}(\mathrm{acac})_{2}$ is seen to be in agreement with the CD spectrum of $\mathrm{Cu}$ $\mathrm{Rpn}$ (acac) $)_{2}$ in $\mathrm{KBr}^{4}$ both with respect to shape and magnitude; this is certainly expected since the only difference between the spectra is that

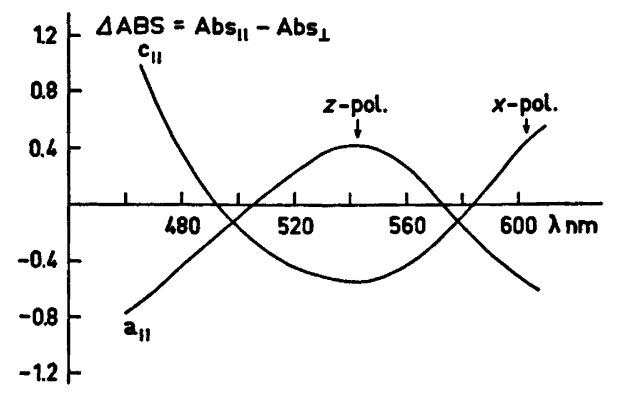

Fig. 1. LD-spectra of $\mathrm{Cu} \mathrm{Rpn}(\mathrm{acac})_{2} . \quad \mathrm{cl}$ is absorption along the c-axis minus absorption along the a-axis of the crystal. The total absorbance of the crystal at $540 \mathrm{~nm}$ is $\sim 6$ measured in the microscopic set-up without use of polarizer and modulator and scaled against a $\mathrm{Cu}$ Rpn(acac) $)_{2}$ solution.

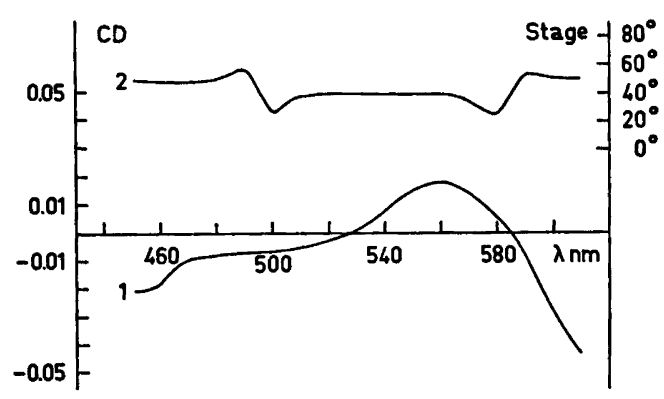

Fig. 2. 1, CD spectrum of Cu Rpn(acac) $)_{2}$ in the crystalline phase. 2, Programming function of the microscope stage to keep $\operatorname{LD}(\lambda)=0$ given relative to stage position $=0^{\circ}$ for the crystal aaxis parallel to the vibration direction of the incoming linearly polarized light.

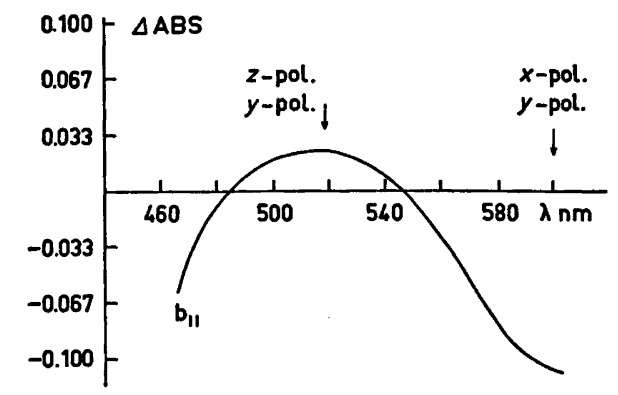

Fig. 3. LD-spectrum of $\mathrm{Cu}$ en(acac) $)_{2}$. $\mathrm{b}_{\|}$is absorption along the b-axis minus absorption along the c-axis of the crystal. The total absorbance of the crystal at $540 \mathrm{~nm}$ is $\sim 6$. 
in $\mathrm{KBr}$ the $\mathrm{LD}$ is zeroed by random orientation of the molecules whereas in the crystals, the $\mathrm{LD}$ is zeroed by rotating the microscope stage.

To check the instrument $\mathrm{LD}$ of $\mathrm{Cu}$ en(acac), was also measured and referring to Table 1 of Ref. 9, it is seen that the band at $620 \mathrm{~nm}$ may be $x$ - and $y$-polarized and the band at $520 \mathrm{~nm}$ $z$ - and $y$-polarized, which is in agreement with the assignments of Olson et al.

\section{DISCUSSION}

Larsen et al. ${ }^{4}$ state that the angle between the two chromophoric parts of $\mathrm{Cu}$ Rpn(acac), in the crystalline phase is $10^{\circ}$ as compared to an estimated angle of $45^{\circ}$ in solution. $A s$ the $\pi \rightarrow \pi^{*}$ transition parts $\left(29000-35000 \mathrm{~cm}^{-1}\right)$ in the solution spectra of $\mathrm{Cu} \mathrm{Rpn}$ (acac) $)_{2}$ and $\mathrm{Cu}$ en(acac) $)_{2}$ are quite similar, we may expect the same magnitude for the angle in Cu en(acac), since the estimation is based purely on the ratio of the dipole strengths of the split components in the $\pi \rightarrow \pi^{*}$ transition band., 18 The angle between the chromophoric parts of $\mathrm{Cu}$ en(acac) $)_{2}$ in the solid state may be calculated from the crystal data and is found to be $4^{\circ}$. Although the symmetry of either molecule cannot be higher than $C_{2}$ (and even $C_{1}$ for $\mathrm{Cu}$ Rpn(acac) $)_{2}$ because of the methyl group on the ethylene bridge), it is advantageous to use either $C_{2 v}$ or $D_{2}$.

Certainly the geometry of the molecules changes on going from solution into the solid form and as this change is towards a planar configuration for both of the two complexes, it is reasonable to assign contributions in $C_{2 v}(x)$. On the other hand, it is still a fact that the molecules are not exactly planar so there may also be contributions to account for in a $D_{2}$ representation. The representations of the valence shell molecular orbitals in $C_{2 v}(x)$ as well as $D_{8}$ are given in Table 3 .

Transitions of $x$ - and $z$-polarizations giving electric transition dipole moments are sought since the Cotton effects under the absorption bands and the $y$-polarized part of the absorption spectrum have been accounted for.", The $z$-polarized transition at $540 \mathrm{~nm}$ (for $\mathrm{Cu}$ Rpn(acac) $)_{2}$ in solution assigned to $(x z) \rightarrow(x y)$ with $x$-polarization and gaining electric transition dipole moment for the rotational strength from the $x$-polarized $\pi \rightarrow \pi^{*}$ transition ${ }^{*}$ ) could
Table 3. Transformations of molecular orbitals in $\mathrm{Cu} \operatorname{Rpn}(\mathbf{a c a c})_{\mathbf{2}}$.

\begin{tabular}{lll}
\hline$C_{2 v}(x)$ & Orbital & $D_{2}$ \\
\hline$a_{2}$ & $4 \pi_{+}=4 \pi_{1}+4 \pi_{2}{ }^{a}$ & $a$ \\
$b_{1}$ & $4 \pi_{-}=4 \pi_{1}-4 \pi_{2}$ & $b_{2}$ \\
$b_{2}$ & $(x y)$ & $b_{1}$ \\
$a_{1}$ & $\left(x^{2}-y^{2}\right)$ & $a$ \\
$a_{2}$ & $(y z)$ & $b_{2}$ \\
$b_{1}$ & $(x z)$ & $b_{2}$ \\
$a_{1}$ & $\left(z^{2}\right)$ & $a$ \\
$b_{1}$ & $3 \pi_{+}=3 \pi_{1}+3 \pi_{2}$ & $b_{1}$ \\
$a_{2}$ & $3 \pi_{-}=3 \pi_{1}-3 \pi_{2}$ & $b_{3}$ \\
$a_{1}$ & $x$ & $b_{2}$ \\
$b_{2}$ & $y$ & $b_{2}$ \\
$b_{1}$ & $z$ & \\
\hline
\end{tabular}

a The two chromophoric parts of the molecules are numbered 1 and 2 respectively in consistency with the numbering in Ref. 6.

very well have its origin in the $(y z) \rightarrow(x y)$ electronic transition, which when accounted for in $C_{2 v}$, has $z$-polarization. This assignment of the $z$-polarized part of band II is furthermore in agreement with the expectation put forward earlier, ${ }^{6,8}$ namely that $3 d_{x z}$ and $3 d_{y x}$ at the copper ion in a planar or nearly planar complex would have almost the same orbital energy; consequently there is no ambiguity in assigning band II partly to $(x z) \rightarrow(x y)$ and partly to $(y z) \rightarrow(x y)$.

In looking for an $x$-polarized electronic transition, the only obvious candidate is found to be the transition $(x y) \rightarrow(4 \pi)$ in $D_{2}$, and therefore the $x$-polarized part of band I may be tentatively assigned to that transition. This certainly presents a problem since the assignment to band III has been given as either $3 \pi \rightarrow(x y)^{19}$ or $(x y) \rightarrow 4 \pi^{20}$ for bis $(\beta$-ketoeno1ate) complexes of copper(II). However, as Cotton et al. state, as possible assignment to band III (the shoulder at $26000 \mathrm{~cm}^{-1}$ in copper(II) bis(acetylacetonate)) could also be $\sigma^{n} \rightarrow \pi^{*}$, an assignment which was discussed further by Jensen and Larsen ' in the sense that the $\sigma^{n} \rightarrow \pi^{*}$ transitions produce excited states with the same symmetries as the $\pi$ excited states thereby making a coupling between the two kinds of transitions possible.

An alternative assignment to band III is the one given by Downing and Urbach (see 
Table 1), but this is in conflict with the assignment given to the $x$-polarized part of band II, and also with the generally accepted notion that band III is not a $d \rightarrow d$ transition.

Therefore, it is seen that accepting the assign. ment $\sigma^{n} \rightarrow \pi^{*}$ of Jensen and Larsen to band III, it is possible to have $(x y) \rightarrow 4 \pi$ at a somewhat lower energy than $23000 \mathrm{~cm}^{-1}$, supporting the above given assignment of $(x y) \rightarrow 4 \pi$ to the $16000 \mathrm{~cm}^{-1} x$-polarized absorption band, an assignment which is furthermore in accordance with the calculations of Cotton et al.

Acknowledgements. The author is most grateful to Professor J. A. Schellman for the opportunity to visit his laboratory on grants from the National Institutes of Health (GM 20195, GM 15423) and the National Science Foundation (GB 41459), and also to Statens Naturvidenskabelige Forskningsråd (511-3785) for providing the travelling funds. The access to the crystal data of Drs. S. Larsen, E. Larsen, E. Røen and K. J. Watson prior to publication, the PVA-film provided by Dr. B. Nordén and the positioning of unit cell axes in actual crystals by Dr. R. Fenna are highly appreciated.

\section{REFERENCES}

1. Hall, D., Rae, A. D. and Waters, T. N. $J$. Chem. Soc. (1963) 5897.

2. Clark, G. R., Hall, D. and Waters, T. N. J. Chem. Soc. $A$ (1968) 223.

3. Hall, D., Morgan, H. J. and Waters, T. N. J. Chem. Soc. A (1966) 677.

4. Larsen, E., Larsen, S., Røen, S. and Watson, K. J. Acta Chem. Scand. A 30 (1976) 125.

5. Downing, R. S. and Urbach, F. L. J. Amer. Chem. Soc. 91 (1969) 5977.

6. Jensen, H. P. and Larsen, E. Acta Chem. Scand. 25 (1971) 1439.

7. Jensen, H. P., Davidsson, A. and Nordén, B. Inorg. Nucl. Chem. Lett. 11 (1975) 67.

8. Cotton, F. A., Harris, C. B. and Wise, J. J. Inorg. Chem. 6 (1967) 909.

9. Olson, C. D., Basu, G. and Linn Belford, R. J. Coord. Chem. 1 (1971) 17.

10 Jones, R. C. J. Opt. Soc. Am. 38 (1948) 671.

11. Gō, N. J. Phys. Soc. Jap. 23 (1967) 88.

12. Troxell, T. C. and Scheraga, H. A. Macromolecules 4 (1971) 519.

13. Hofrichter, H. J. and Schellman, J. A. Jerusalem Symposia on Quantum Chemistry and Biochemistry $V$. The Israel Academy of Sciences and Humanities, Jerusalem, 1973.

14. Kemp. J. J. Opt. Soc. Am. 59 (1969) 950.

15. Shurcliff, W. A. Polarized Light, Harvard Univ. Press., Cambridge, Mass. 1962.

16. Schmieder, R. W. J. Opt. Soc. Am. 59 (1969) 297.
17. Nordén, B. Acta Chem. Scand. 27 (1973) 4021.

18. Larsen, E. Acta Chem. Scand. 23 (1969) 2158.

19. Jørgensen, C. K. Acta Chem. Scand. 16 (1962) 2406.

20. Cotton, F. A. and Wise, J. J. Inorg. Chem. 6 (1967) 917.

Received June 9, 1975. 